89.

\title{
ON THE ATTRACTION OF ELLIPSOIDS (JACOBI'S METHOD).
}

[From the Cambridge and Dublin Mathematical Journal, vol. v. (1850), pp. 217-226.]

IN a letter published in 1846 in Liouville's Journal (t. XI. p. 341) Jacobi says, "Il y a quatorze ans, je me suis posé le problème de chercher l'attraction d'un ellipsoïde homogène exercée sur un point extérieur quelconque par une méthode analogue à celle employée par Maclaurin par rapport aux points situés dans les axes principaux. J'y suis parvenu par trois substitutions consécutives. La première est une transformation de coordonnées; par la seconde le radical $\sqrt{ }\left(1-m^{2} \sin ^{2} \beta \cos ^{2} \psi-n^{2} \sin ^{2} \beta \sin ^{2} \psi\right)$ qui entre dans la double intégrale transformée est rendu rationnel au moyen de la double substitution

$$
m \sin \beta \cos \psi=\sin \eta \cos \theta, \quad m \sin \beta \sin \psi=\sin \eta \sin \theta ;
$$

la troisième est encore une transformation de coordonnées. La recherche du sens géométrique de ces trois substitutions m'a conduit à approfondir la théorie des surfaces confocales par rapport auxquelles je découvris quantité de beaux théorèmes dont je communiquai quelques-uns des principaux à M. Steiner. Considérons l'ellipsoïde confocal mené par le point attiré $P$ et le point $p$ de l'ellipsoïde proposé, conjugué à $P$. Soient $Q$ et $q$ deux autres points conjugués quelconques situés respectivement sur l'ellipsoïde extérieur et intérieur. Menons de $P$ un premier cône tangent à l'ellipsoïde intérieur, de $p$ un second cône tangent à l'ellipsoïde extérieur. Ce dernier, tout imaginaire qu'il est, a ses trois axes réels (ainsi que ses deux droites focales). La première substitution ramène les axes de l'ellipsoïde à ceux du premier cône (c'est la substitution employée par Poisson, mais que j'avais antérieurement traitée et même étendue à un nombre quelconque de variables dans le mémoire De binis Functionibus homogeneis \&c. [Crelle, t. xII. (1834) pp. 1-69]). Par la seconde substitution les angles que la droite $P q$ forme avec les axes du premier cône sont ramenés aux angles que la droite $p Q$ forme avec les axes du second. Par la dernière substitution, on retourne de ces axes aux axes de l'ellipsoïde. La seconde substitution répond à un théorème de géométrie remarquable, savoir que 'Les cosinus des angles que la droite $P q$ forme avec deux des axes du premier cône sont en raison constante avec les cosinus des angles que la droite 
$p Q$ forme avec deux des axes du second cône; ces deux axes sont les tangents situés respectivement dans les sections de plus grande et de moindre courbure de chaque ellipsoïde, le troisième axe étant la normale à l'ellipsoïde.' Tout cela semble difficile à établir par la synthèse."

The object of this paper is to develope the above method of finding the attraction of an ellipsoid.

Consider an exterior ellipsoid, the squared semiaxes of which are $f+u, g+u, h+u$; and an interior ellipsoid, the squared semiaxes of which are $f+\bar{u}, g+\bar{u}, h+\bar{u}$. Let $u, p, q$ be the elliptic coordinates of a point $P$ on the exterior ellipsoid, the elliptic coordinates of the corresponding point $\bar{P}$ on the interior ellipsoid will be $\bar{u}, p$, $q$, and if $a, b, c$ and $\bar{a}, \bar{b}, \bar{c}$ represent the ordinary coordinates of these points (the principal axes being the axes of coordinates), we have

$$
\begin{aligned}
& a^{2}=\frac{(f+u)(f+q)(f+r)}{(f-g)(f-h)}, \\
& b^{2}=\frac{(g+u)(g+q)(g+r)}{(g-h)(g-f)}, \\
& c^{2}=\frac{(h+u)(h+q)(h+r)}{(h-g)(h-f)},
\end{aligned}
$$

$$
\begin{aligned}
& \bar{a}^{2}=\frac{(f+\bar{u})(f+q)(f+r)}{(f-g)(f-h)}, \\
& \bar{b}^{2}=\frac{(g+\bar{u})(g+q)(g+r)}{(g-h)(g-f)}, \\
& \bar{c}^{2}=\frac{(h+\bar{u})(h+q)(h+r)}{(h-f)(h-g)} .
\end{aligned}
$$

I form the systems of equations

$$
\begin{aligned}
a_{1}{ }^{2}=\frac{(u+f)(u+g)(u+h)}{(u-q)(u-r)}, & & \bar{a}_{1}^{2}=\frac{(\bar{u}+f)(\bar{u}+g)(\bar{u}+h)}{(\bar{u}-q)(\bar{u}-r)}, \\
b_{1}{ }^{2}=\frac{(q+f)(q+g)(q+h)}{(q-r)(q-u)}, & & \bar{b}_{1}^{2}=\frac{(q+f)(q+g)(q+h)}{(\bar{q}-r)(q-\bar{u})}, \\
c_{1}{ }^{2}=\frac{(r+f)(r+g)(r+h)}{(r-u)(r-q)}, & & \bar{c}_{1}^{2}=\frac{(r+f)(r+g)(r+h)}{(r-\bar{u})(r-q)} . \\
\alpha=\frac{a_{1} a}{f+u}, & \beta=\frac{a_{1} b}{g+u}, & \gamma=\frac{a_{1} c}{h+u}, \\
\alpha^{\prime}=\frac{b_{1} a}{f+q}, & \beta^{\prime}=\frac{b_{1} b}{g+q}, & \gamma^{\prime}=\frac{b_{1} c}{h+q}, \\
\alpha^{\prime \prime}=\frac{c_{1} a}{f+r}, & \beta^{\prime \prime}=\frac{c_{1} b}{g+r}, & \gamma^{\prime \prime}=\frac{c_{1} c}{h+r}, \\
\bar{\alpha}=\frac{\bar{a} \bar{a}}{f+\bar{u}}, & \bar{\beta}=\frac{\overline{a_{1}} \bar{b}}{g+\bar{u}}, & \bar{\gamma}=\frac{\bar{a}_{1} \bar{c}}{h+\bar{u}}, \\
\bar{\alpha}^{\prime}=\frac{\bar{b}_{1} \bar{a}}{f+q}, & \bar{\beta}^{\prime}=\frac{\bar{b}_{1} \bar{b}}{g+q}, & \overline{\gamma^{\prime}}=\frac{\bar{b}_{1} \bar{c}}{h+q}, \\
\bar{\alpha}^{\prime \prime}=\frac{\bar{c}_{1} \bar{a}}{f+r}, & \overline{\beta^{\prime \prime}}=\frac{\bar{c}_{1} \bar{b}}{g+r}, & \overline{\gamma^{\prime \prime}}=\frac{\bar{c}_{1} \bar{c}}{h+r} .
\end{aligned}
$$


And then writing

$$
\begin{array}{l|l}
X=\alpha X_{1}+\alpha^{\prime} Y_{1}+\alpha^{\prime \prime} Z_{1}, & \bar{X}=\bar{\alpha} \bar{X}_{1}+\bar{\alpha}^{\prime} \bar{Y}_{1}+\bar{\alpha}^{\prime \prime} \bar{Z}_{1}, \\
Y=\beta X_{1}+\beta^{\prime} Y_{1}+\beta^{\prime \prime} Z_{1}, & \bar{Y}=\bar{\beta} \bar{X}_{1}+\bar{\beta}^{\prime} \bar{Y}_{1}+\bar{\beta}^{\prime \prime} \bar{Z}_{1}, \\
Z=\gamma X_{1}+\gamma^{\prime} Y_{1}+\gamma^{\prime \prime} Z_{1}, & \bar{Z}=\bar{\gamma} \bar{X}_{1}+\bar{\gamma}^{\prime} \bar{Y}_{1}+\bar{\gamma}^{\prime \prime} \bar{Z}_{1},
\end{array}
$$

if $X, Y, Z$ are the cosines of the inclinations of a line $P \bar{Q}$ to the principal axes of the ellipsoids, $X_{1}, Y_{1}, Z_{1}$ will be the cosines of the inclinations of this line to the principal axes' of the cone having $P$ for its vertex, and circumscribed about the interior ellipsoid. In like manner, $X, \bar{Y}, \bar{Z}$ being the cosines of the inclinations of a line $\bar{P} Q$ to the principal axes of the ellipsoids, $\bar{X}_{1}, \bar{Y}_{1}, \bar{Z}_{1}$ will be the cosines of the inclinations of this line to the principal axes of the cone having $\bar{P}$ for its vertex and circumscribed about the exterior ellipsoid. Assuming that the points $Q, \bar{Q}$ are situated upon the exterior and interior ellipsoids respectively, suppose that $X_{1}, Y_{1}, Z_{1}$ and $\bar{X}_{1}, \bar{Y}_{1}, \bar{Z}_{1}$ are connected by the equivalent systems of equations,

$$
\begin{aligned}
& X_{1}=\sqrt{ }(u-\bar{u}) \sqrt{ }\left(\frac{\bar{X}_{1}^{2}}{u-\bar{u}}+\frac{\bar{Y}_{1}^{2}}{u-q}+\frac{\bar{Z}_{1}^{2}}{u-r}\right), \\
& Y_{1}=\sqrt{\left(\frac{\bar{u}-q}{u-q}\right) \bar{Y}_{1},} \\
& Z_{1}=\sqrt{\left(\frac{\bar{u}-r}{u-r}\right) \bar{Z}_{1},} \\
& \bar{X}_{1}=\sqrt{ }(\bar{u}-u) \sqrt{ }\left(\frac{X_{1}^{2}}{\bar{u}-u}+\frac{Y_{1}^{2}}{\bar{u}-q}+\frac{Z_{1}^{2}}{\bar{u}-r}\right), \\
& Y_{1}=\sqrt{\left(\frac{u-q}{\bar{u}-q}\right) Y_{1},} \\
& \bar{Z}_{1}=\sqrt{ }\left(\frac{u-r}{\bar{u}-r}\right) Z_{1} ;
\end{aligned}
$$

then it will presently be shown that the points $Q, \bar{Q}$ are corresponding points, which will prove the geometrical theorem of Jacobi. Before proceeding further it will be convenient to notice the formulæ

$$
\begin{aligned}
& 1-\frac{a^{2}}{f+\bar{u}}-\frac{b^{2}}{g+\bar{u}}-\frac{c^{2}}{h+\bar{u}}=\frac{\bar{u}-u}{\bar{a}_{1}^{2}}, \\
& \frac{X a}{f+\bar{u}}+\frac{Y b}{g+\bar{u}}+\frac{Z c}{h+\bar{u}}=\frac{\bar{u}-u}{\bar{a}_{1}^{2}}\left(\frac{X_{1} a_{1}}{\bar{u}-u}+\frac{Y_{1} b_{1}}{\bar{u}-q}+\frac{Z_{1} c_{1}}{\bar{u}-r}\right), \\
& \left(\frac{X a}{f+\bar{u}}+\frac{Y b}{g+\bar{u}}+\frac{Z c}{h+\bar{u}}\right)^{2}+\frac{\bar{u}-u}{\bar{a}_{1}^{2}}\left(\frac{X^{2}}{f+\bar{u}}+\frac{Y^{2}}{g+\bar{u}}+\frac{Z^{2}}{h+\bar{u}}\right) \\
& =\frac{\bar{u}-u}{\bar{a}_{1}^{2}}\left(\frac{X_{1}^{2}}{\bar{u}-u}+\frac{Y_{1}^{2}}{\bar{u}-q}+\frac{Z_{1}^{2}}{\bar{u}-r}\right)=\frac{\bar{X}_{1}^{2}}{\bar{a}_{1}^{2}} ;
\end{aligned}
$$

C. 
and the corresponding ones

$$
\begin{aligned}
& 1-\frac{\bar{a}^{2}}{f+u}-\frac{\bar{b}^{2}}{g+u}-\frac{\bar{c}^{2}}{h+u}=\frac{u-\bar{u}}{a_{1}^{2}}, \\
& \frac{\bar{X} \bar{a}}{\bar{f}+u}+\frac{\bar{Y} \bar{b}}{g+u}+\frac{\bar{Z} \bar{c}}{h+\bar{u}}=\frac{u-\bar{u}}{a_{1}{ }^{2}}\left(\frac{\bar{X}_{1} \bar{a}_{1}}{u-\bar{u}}+\frac{\bar{Y}_{1} \bar{b}_{1}}{u-q}+\frac{\bar{Z}_{1} \bar{c}_{1}}{u-r}\right), \\
& \begin{aligned}
\left(\frac{\bar{X} \bar{a}}{f+u}+\frac{\bar{Y} \bar{b}}{g+u}+\frac{\bar{Z} \bar{c}}{h+u}\right)^{2}+\frac{u-\bar{u}}{a_{1}{ }^{2}} & \left(\frac{\bar{X}^{2}}{f+u}+\frac{\bar{Y}^{2}}{g+u}+\frac{\bar{Z}^{2}}{h+u}\right) \\
& =\frac{u-\bar{u}}{a_{1}{ }^{2}}\left(\frac{\bar{X}_{1}^{2}}{u-\bar{u}}+\frac{\bar{Y}_{1}^{2}}{u-q}+\frac{\bar{Z}_{1}^{2}}{u-r}\right)=\frac{X_{1}^{2}}{a_{1}^{2}} .
\end{aligned}
\end{aligned}
$$

The coordinates of the point $\bar{Q}$ are obviously $a+\rho X, b+\rho Y, c+\rho Z$ (where $\rho=P \bar{Q}$ ); substituting these values in the equation of the interior ellipsoid, we obtain

$$
\rho^{2}\left(\frac{X^{2}}{f+\bar{u}}+\frac{Y^{2}}{g+\bar{u}}+\frac{Z^{2}}{h+\bar{u}}\right)+2 \rho\left(\frac{X a}{f+\bar{u}}+\frac{Y b}{g+\bar{u}}+\frac{Z c}{h+\bar{u}}\right)+\left(\frac{a^{2}}{f+\bar{u}}+\frac{b^{2}}{g+\bar{u}}+\frac{c^{2}}{h+\bar{u}}-1\right)=0:
$$

reducing the coefficients of this equation by the formulæ first given, and omitting a factor $\frac{\bar{u}-u}{\bar{a}_{1}^{2}}$, we obtain

$$
\left\{\frac{\bar{a}_{1}^{2} \bar{X}^{2}}{(\bar{u}-u)^{2}}-\left(\frac{X_{1} a_{1}}{\bar{u}-u}+\frac{Y_{1} b_{1}}{\bar{u}_{1}-q}+\frac{Z_{1} c_{1}}{\bar{u}-r}\right)^{2}\right\} \rho^{2}+2 \rho\left(\frac{X_{1} a_{1}}{\bar{u}-u}+\frac{Y_{1} b_{1}}{\bar{u}-q}+\frac{Z_{1} c_{1}}{\bar{u}-r}\right)-1=0,
$$

that is,

$$
\frac{\bar{a}_{1}^{2} \bar{X}_{1}^{2}}{(\bar{u}-u)^{2}} \rho^{2}=\left\{\rho\left(\frac{X_{1} a_{1}}{\bar{u}-u}+\frac{Y_{1} b_{1}}{\bar{u}-q}+\frac{Z_{1} c_{1}}{\bar{u}-r}\right)-1\right\}^{2},
$$

or

$$
\rho=\frac{1}{\frac{X_{1} a_{1}-\bar{X}_{1} \bar{a}_{1}}{\bar{u}-u}+\frac{Y_{1} a_{1}}{\bar{u}-q}+\frac{Z_{1} c_{1}}{\bar{u}-r}} ;
$$

which is easily transformed into

$$
\rho=\frac{\bar{u}-u}{a_{1} X_{1}-\bar{a}_{1} \bar{X}_{1}+\frac{(f+u) b_{1} Y_{1}-(f+\bar{u}) \bar{b}_{1} \bar{Y}_{1}}{f+q}+\frac{(f+u) c_{1} Z_{1}-(f+\bar{u}) \bar{c}_{1} \bar{Z}_{1}}{f+r}}:
$$

and this form remaining unaltered when $u$ and $\bar{u}$ are interchanged, it follows that if $\bar{P} Q=\bar{\rho}$, then $\rho=\bar{\rho}$, which is a known theorem. The value of $\rho$ or $\bar{\rho}$ may however be expressed in a yet simpler form; for, considering the expression

$$
\begin{aligned}
\frac{X}{\sqrt{ }(f+\bar{u})}- & \frac{\bar{X}}{\sqrt{ }(f+u)}=\frac{a}{\sqrt{ }(f+\bar{u})}\left\{\frac{a_{1} X_{1}}{f+u}+\frac{b_{1} Y_{1}}{f+q}+\frac{c_{1} Z_{1}}{f+r}\right\}-\frac{\bar{a}}{\sqrt{ }(f+u)}\left\{\frac{\bar{a}_{1} \bar{X}_{1}}{f+\bar{u}}+\frac{\bar{b}_{1} \bar{Y}_{1}}{f+q}+\frac{\bar{c}_{1} \bar{Z}_{1}}{f+r}\right\} \\
=\frac{-1}{\bar{u}-u} & \left\{\frac{a}{\sqrt{ }(f+u)}-\frac{\bar{a}}{\sqrt{ }(f+u)}\right\} \\
& \times\left\{a_{1} X_{1}-\bar{a}_{1} \bar{X}_{1}+\frac{(f+u) b_{1} Y_{1}-(f+\bar{u}) \bar{b}_{1} \bar{Y}_{1}}{f+q}+\frac{(f+u) c_{1} Z_{1}-(f+\bar{u}) \bar{c}_{1} \bar{Z}_{1}}{f+r}\right\},
\end{aligned}
$$


we see that

$$
\frac{X}{\sqrt{ }(f+\bar{u})}-\frac{\bar{X}}{\sqrt{ }(f+u)}=-\frac{1}{\rho}\left(\frac{a}{\sqrt{ }(f+\bar{u})}-\frac{\bar{a}}{\sqrt{ }(f+u)}\right),
$$

and similarly

$$
\begin{aligned}
& \frac{Y}{\sqrt{ }(f+\bar{u})}-\frac{\bar{Y}}{\sqrt{ }(g+u)}=-\frac{1}{\rho}\left(\frac{b}{\sqrt{ }(g+\bar{u})}-\frac{\bar{b}}{\sqrt{ }(g+u)}\right) \\
& \frac{Z}{\sqrt{(h+\bar{u})}}-\frac{\bar{Z}}{\sqrt{ }(h+u)}=-\frac{1}{\rho}\left(\frac{c}{\sqrt{ }(h+\bar{u})}-\frac{\bar{c}}{\sqrt{ }(h+u)}\right)
\end{aligned}
$$

which are in fact the equations which express that $Q$ and $\bar{Q}$ are corresponding points.

It is proper to remark that supposing, as we are at liberty to do, that $P, \bar{P}$ are situate in corresponding octants of the two ellipsoids, then if the curve of contact of the circumscribed cone having $P$ for its vertex divide the surface of the interior ellipsoid into two parts $\bar{M}, \bar{N}$, of which the former lies contiguous to $\bar{P}$ : also if the curve of intersection of the tangent plane at $\bar{P}$ divide the surface of the exterior ellipsoid into two parts $M, N$, of which $M$ lies contiguous to the point $P$; then the different points of $M, \bar{M}$ correspond to each other, as do also the different points of $N, \bar{N}$.

We may now pass to the integral calculus problem. The Attraction parallel to the axis of $x$ is

$$
A=\int \frac{x d x d y d z}{\left(x^{2}+y^{2}+z^{2}\right)^{\frac{3}{2}}}
$$

the limits of the integration being given by

$$
\frac{(x+a)^{2}}{f+\bar{u}}+\frac{(y+b)^{2}}{g+\bar{u}}+\frac{(z+c)^{2}}{h+\bar{u}}=1
$$

or putting

$$
x=r X, \quad y=r Y, \quad z=r Z,
$$

where $X, Y, Z$ have the same signification, as before, we have

$$
d x d y d z=r^{2} d r d S
$$

and then

$$
A=\int X d r d S=\int \rho X d S
$$

where $\rho$ has the same signification as before: it will be convenient to leave the formula in this form, rather than to take at once the difference of the two values of $\rho$, but of course the integration is as in the ordinary methods to be performed so as to extend to the whole volume of the ellipsoid. The expression $d S$ denotes the differen- 
tial of a spherical surface radius unity, and if $\theta, \phi$ are the parameters by which the position of $\rho$ is determined, we have

$$
d S=\left|\begin{array}{ccc}
X, & Y, & Z \\
\frac{d X}{d \theta}, & \frac{d Y}{d \theta}, & \frac{d Z}{d \theta} \\
\frac{d X}{d \phi}, & \frac{d Y}{d \phi}, & \frac{d Z}{d \phi}
\end{array}\right| d \theta d \phi
$$

In the present case

$$
d S=d S_{1}=\left|\begin{array}{ccc}
X_{1}, & Y_{1}, & Z_{1} \\
\frac{d X_{1}}{d \bar{Y}_{1}}, & \frac{d Y_{1}}{d \bar{Y}_{1}}, & \frac{d Z_{1}}{d \bar{Y}_{1}} \\
\frac{d X_{1}}{d \bar{Z}_{1}}, & \frac{d Y_{1}}{d \bar{Z}_{1}}, & \frac{d Z_{1}}{d \bar{Z}_{1}}
\end{array}\right| d \bar{Y}_{1} d \bar{Z}_{1}
$$

or from the values of $X_{1}, Y_{1}, Z_{1}$ in terms of $\bar{X}_{1}, \bar{Y}_{1}, \bar{Z}_{1}$ \{observing that $\bar{X}_{1}$ must be replaced by its value $\left.\sqrt{ }\left(1-\bar{Y}_{1}^{2}-\bar{Z}_{1}^{2}\right)\right\}$ we deduce

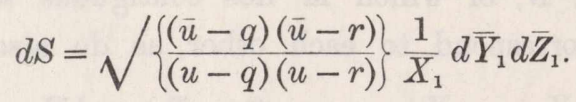

But $d \bar{S}=d \bar{S}_{1}=\frac{1}{\bar{X}_{1}} d \bar{Y}_{1} d \bar{Z}_{1}$,

whence

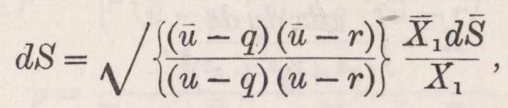

which shows that the corresponding elements of the spheres whose centres are $P, \bar{P}$, projected upon the tangent planes at $P$ and $\bar{P}$ respectively, are in a constant ratio. It may be noticed also that if $\mu, \bar{\mu}$ are the masses of the ellipsoids, the ratio in question

$$
=\sqrt{\left\{\frac{(\bar{u}-q)(\bar{u}-r)}{(u-q)(u-r)}\right\}=\frac{\bar{\mu} a_{1}}{\mu \bar{a}_{1}} .}
$$

We have thus

$$
\left.A=\sqrt{\left\{\frac{(\bar{u}-q)(\bar{u}-r)}{(u-q)(u-r)}\right\}}\right\} \frac{\bar{\rho} \bar{X}_{1} X d \bar{S}}{X_{1}}
$$

that is

$$
A=\sqrt{\left\{\frac{(\bar{u}-q)(\bar{u}-r)}{(u-q)(u-r)}\right\}} \int \frac{\bar{\rho}\left(\alpha X_{1}+\alpha^{\prime} Y_{1}+\alpha^{\prime \prime} Z_{1}\right) \overline{X_{1}} d \bar{S}}{X_{1}} .
$$

The value which it will be convenient to use for $\bar{\rho}$ is that derived from the equation

$$
\bar{\rho}^{2}\left(\frac{\bar{X}^{2}}{f+u}+\frac{\bar{Y}^{2}}{g+u}+\frac{\bar{Z}^{2}}{h+u}\right)+2 \bar{\rho}\left(\frac{\bar{X} \bar{a}}{f+u}+\frac{\bar{Y} \bar{b}}{g+u}+\frac{\bar{Z}^{\prime} c}{h+u}\right)+\frac{\bar{u}-u}{a_{x}{ }^{2}}=0,
$$


with only the transformation of expressing the radical in terms of $X_{1}$, viz.

$$
\rho=\frac{\frac{\bar{X} \bar{a}}{f+u}+\frac{\bar{Y} \bar{b}}{g+u}+\frac{\bar{Z} \bar{c}}{h+u}+\frac{1}{a_{1}} X_{1}}{\frac{\bar{X}^{2}}{f+u}+\frac{\bar{Y}^{2}}{g+u}+\frac{\bar{Z}^{2}}{h+u}}
$$

substituting these values and observing that $Y_{1}$ and $Z_{1}$ are rational functions of $\bar{X}, \bar{Y}$, and $\bar{Z}$, but that $\bar{X}_{1}$ is a radical, and that in order to extend the integration to the whole ellipsoid, the values corresponding to the opposite signs of $X_{1}$ will require to be added, the quantity to be integrated (omitting for the moment the exterior constant factor) is

$$
=\frac{\left\{\alpha\left(\frac{\bar{X} \bar{a}}{f+u}+\frac{\bar{Y} \bar{b}}{g+u}+\frac{\bar{Z} \bar{c}}{h+u}\right)+\frac{1}{a_{1}}\left(\alpha^{\prime} Y_{\mathrm{i}}+\alpha^{\prime \prime} Z_{1}\right)\right\} \bar{X}_{1} d \bar{S}}{\frac{\bar{X}^{2}}{f+u}+\frac{\overline{Y^{2}}}{g+u}+\frac{\bar{Z}^{2}}{h+u}},
$$

the integration to be extended over the spherical area $\bar{S}$. Consider the quantity within \{\} , this is

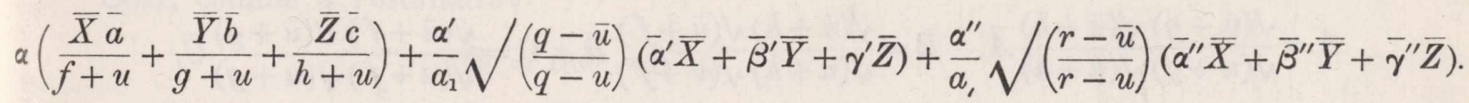

The coefficients of $\bar{Y}$ and $\bar{Z}$ vanish, in fact that of $\bar{Y}$ is

$$
\begin{aligned}
& \frac{a_{1} a}{f+u} \frac{\bar{b}}{g+u}+\frac{b_{1} a}{a_{1}(f+q)} \sqrt{\left(\frac{q-\bar{u}}{q-u}\right) \frac{\bar{b}_{1} \bar{b}}{g+q}+\frac{c_{1} a}{a_{1}(f+r)} \sqrt{ }\left(\frac{r-\bar{u}}{r-u}\right) \frac{\bar{c}_{1} \bar{b}}{g+r}} \\
= & \frac{a \bar{b}}{a_{1}}\left\{\frac{a_{1}{ }^{2}}{(f+u)(g+u)}+\frac{b_{1} \bar{b}_{1}}{(f+q)(g+q)} \sqrt{\left(\frac{q-\bar{u}}{q-u}\right)+\frac{c_{1} \bar{c}_{1}}{(f+r)(g+r)}} /\left(\frac{r-\bar{u}}{r-u}\right)\right\} \\
= & \frac{a b_{1}}{a_{1}}\left\{\frac{a_{1}{ }^{2}}{(f+u)(g+u)}+\frac{b_{1}{ }^{2}}{(f+q)(g+q)}+\frac{c_{1}{ }^{2}}{(f+r)(g+r)}\right\}=0 ;
\end{aligned}
$$

and similarly for the coefficient of $\bar{Z}$.

The coefficient of $\bar{X}$ is in like manner shown to be

$$
\frac{a \bar{a}}{a_{1}}\left\{\frac{a_{1}^{2}}{(f+u)^{2}}+\frac{b_{1}^{2}}{(f+q)^{2}}+\frac{c_{1}^{2}}{(f+r)^{2}}\right\}=\frac{a \bar{a}}{a_{1}} \frac{(f-g)(f-h)}{(f+u)(f+q)(f+r)}=\frac{a \bar{a}}{a^{2} a_{1}}=\frac{\bar{a}}{a a_{1}}
$$

or the quantity in question is simply

$$
\frac{\bar{a}}{a a_{1}} \bar{X}
$$


Multiplying this by $\bar{X}_{1},=\bar{\alpha} X+\bar{\alpha}^{\prime} \bar{Y}+\bar{\alpha}^{\prime \prime} \bar{Z}$, the terms containing $\bar{X} \bar{Y}, \bar{X} \bar{Z}$ vanish after the integration, and we need only consider the term $\frac{\bar{a} \bar{\alpha}}{a a_{1}} \bar{X}^{2}$, or what is the same $\frac{\bar{a}^{2} \bar{\alpha}_{1}}{a a_{1}(f+\bar{u})} \bar{X}^{2}$. Hence

$$
\left.A=\sqrt{\left\{\frac{(\bar{u}-q)(\bar{u}-r)}{(u+q)(u-r)}\right\}}\right\} \frac{\bar{a}^{2} \bar{a}_{1}}{a a_{1}(f+\bar{u})} \int \frac{\bar{X}^{2} d \bar{s}}{\frac{\bar{X}^{2}}{f+u}+\frac{\bar{Y}^{2}}{g+u}+\frac{\bar{Z}^{2}}{h+u}}
$$

The value of the corresponding function $\bar{A}$ (that is, the attraction of the exterior ellipsoid upon $\bar{P})$ is

$$
\bar{A}=\frac{\bar{a}}{f+u} \int \frac{\bar{X}^{2} d \bar{S}}{\frac{\bar{X}^{2}}{f+u}+\frac{\bar{Y}^{2}}{g+u}+\frac{\bar{Z}^{2}}{h+u}},
$$

the limits being the same, whence

$$
A \div \bar{A}=\sqrt{ }\left\{\frac{(\bar{u}-q)(\bar{u}-r)}{(u-q)(u-r)}\right\} \frac{f+u}{f+\bar{u}} \frac{\bar{a} \bar{a}_{1}}{a a_{1}}=\frac{\sqrt{ }(\bar{u}+g) \sqrt{ }(\bar{u}+h)}{\sqrt{ }(u+g) \sqrt{ }(u+h)}
$$

or we have

$$
A=\frac{\sqrt{ }(\bar{u}+g) \sqrt{ }(\bar{u}+h)}{\sqrt{ }(u+g) \sqrt{ }(u+h)} \bar{A}, \quad B=\frac{\sqrt{ }(\bar{u}+h) \sqrt{ }(\bar{u}+f)}{\sqrt{ }(u+h) \sqrt{ }(\bar{u}+f)} \bar{B}, \quad C=\frac{\sqrt{ }(\bar{u}+f) \sqrt{ }(\bar{u}+g)}{\sqrt{ }(u+f) \sqrt{ }(u+g)} \bar{C},
$$

formulæ which constitute in fact Ivory's theorem.

Let $K, \bar{K}$ denote the attractions in the directions of the normals at $P, \bar{P}$, we have

$$
\begin{aligned}
K & =\frac{\bar{\mu} a_{1}}{\mu \bar{a}_{1}} \int \bar{X}_{1} d \bar{S}, \quad \bar{K}=\int \bar{X}_{1} d \bar{S}, \\
K & =\frac{\bar{\mu} a_{1}}{\mu \bar{a}_{1}} K
\end{aligned}
$$

and it is important to remark that this is true not only for the entire ellipsoids; but if $\mathfrak{A} \mathfrak{A}, \mathfrak{I}$ denote the attractions of the cones standing on the portions $\bar{M}, \bar{N}$ of the surface of the interior ellipsoid, and $\overline{\sqrt[3]{ } \boldsymbol{l}}, \overline{\sqrt{2}}$ the attractions of the portions of the exterior ellipsoid bounded by the tangent plane at $\bar{P}$, and the portions $M, N$ of the surface of the exterior ellipsoid, then

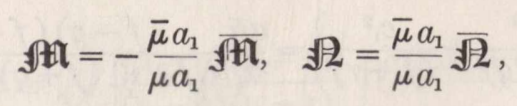

where obviously

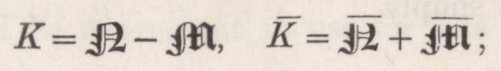

this theorem is so far as I am aware new. 